\title{
Safety at the Time of the COVID-19 Pandemic: How to Keep Our Oncology Patients and Healthcare Workers Safe
}

Pelin Cinar, MD, MS; Timothy Kubal, MD, MBA²; Alison Freifeld, MD³; Asmita Mishra, MD²; Lawrence Shulman, MD; James Bachman, MPA ${ }^{5}$; Rafael Fonseca, MD6; Hope Uronis, MD, MHS7; Dori Klemanski, DNP8;

Kim Slusser, MSN, CHPN, NEA-BC ${ }^{9}$; Matthew Lunning, $\mathrm{DO}^{3}$; and Catherine Liu, MD ${ }^{10,11}$

\begin{abstract}
The novel coronavirus, SARS-CoV-2, was first detected as a respiratory illness in December 2019 in Wuhan City, China. Since then, coronavirus disease 2019 (COVID-19) has impacted every aspect of our lives worldwide. In a time when terms such as social distancing and flattening the curve have become a part of our vernacular, it is essential that we understand what measures can be implemented to protect our patients and healthcare workers. Undoubtedly, healthcare providers have had to rapidly alter care delivery models while simultaneously acknowledging the crucial unknowns of how these changes may affect clinical outcomes. This special feature reviews strategies on how to mitigate transmission of COVID-19 in an effort to reduce morbidity and mortality associated with the disease for patients with cancer without infection, for patients with cancer with COVID-19 infection, and for the healthcare workers caring for them, while continuing to provide the best possible cancer care. [Editor's Note: This article includes the most current information available at time of publication; however, recommendations regarding public safety and practice may change rapidly in this situation. Individuals should get the most up to date information from the CDC website.]
\end{abstract}

J Natl Compr Canc Netw 2020;18(5):504-509 doi: $10.6004 /$ jnccn.2020.7572

\footnotetext{
${ }^{1}$ Division of Medical Oncology, Department of Medicine, UCSF Helen Diller Family Comprehensive Cancer Center, California; ${ }^{2}$ Moffitt Cancer Center, Tampa, Florida; ${ }^{3}$ Fred \& Pamela Buffett Cancer Center, Omaha, Nebraska; ${ }^{4}$ Abramson Cancer Center at the University of Pennsylvania, Philadelphia, Pennsylvania; ${ }^{5}$ University of Colorado Cancer Center, Aurora, Colorado; ${ }^{6}$ Mayo Clinic Cancer Center, Phoenix, Arizona; ${ }^{7}$ Duke Cancer Institute, Durham, North Carolina; ${ }^{8}$ Ohio State Comprehensive Cancer Center - James Cancer Hospital and Solove Research Institute, Columbus, Ohio; ${ }^{9}$ Yale Cancer Center/Smilow Cancer Hospital, New Haven, Connecticut; and ${ }^{10}$ Vaccine and Infectious Diseases Division, Fred Hutchinson Cancer Research Center, and ${ }^{11}$ Division of Allergy and Infectious Diseases, Department of Medicine, University of Washington, Seattle, Washington.
}

The novel coronavirus, SARS-CoV-2, was first detected as a respiratory illness in December 2019 in Wuhan City, China. Since detection, the disease caused by SARS-CoV2, known as coronavirus disease 2019 (COVID-19), has impacted every aspect of our lives worldwide. On March 11,2020 , COVID-19 was categorized as a pandemic when $>118,000$ cases were diagnosed globally. ${ }^{1}$ As of April 1, 2020 , the number of confirmed cases increased to 932,605 with 46,809 deaths. ${ }^{2}$ The first confirmed case in the United States was reported on January 20, 2020, in Snohomish County, Washington. At a time when terms such as social distancing and flattening the curve have become a part of our vernacular, it is essential that we understand what measures can be implemented to protect patients and healthcare workers. China, Italy, and Spain have reported that $3.8 \%$ to $10 \%$ of confirmed COVID-19-positive cases were healthcare workers. ${ }^{3-5}$ Undoubtedly, healthcare providers have had to rapidly alter care delivery models while simultaneously acknowledging the crucial unknowns of how these changes may affect clinical outcomes. In this special feature, we review strategies for mitigating transmission of COVID19 in an effort to reduce morbidity and mortality associated with the disease for patients with cancer without infection, for patients with cancer with COVID-19 infection, and for the healthcare workers caring for them, while continuing to provide the best possible cancer care.

\section{Patient Safety}

Reports from the Chinese experience have shown that patients with cancer may have an increased risk of contracting and developing complications from COVID$19 .{ }^{6,7}$ A retrospective study of 1,524 patients with cancer, half of whom were not on active therapy, showed a higher risk of COVID-19 (odds ratio [OR], 2.31; 95\% CI, 1.89-3.02) compared with the community. ${ }^{7}$ This risk was small and was likely due to frequent visits to the hospital for treatment. In a prospective cohort study of 1,590 
COVID-19 cases, 18 patients (1\%) had a history of cancer, with lung cancer most frequently reported. Patients with cancer were more likely to experience clinically severe events, defined as treatment in an intensive care unit or death, compared with those without cancer $(39 \%$ vs $8 \%$; $P=.0003) .^{6}$ Additionally, those who underwent chemotherapy or surgery in the past month had a greater risk of experiencing clinically severe events compared with those who had not received recent treatment (OR, 5.34; 95\% CI, 1.80-16.18; $P=.0026$ ). The risk also appears to be higher in patients with $>1$ chronic medical condition. ${ }^{6}$ Even with the intrinsic limitations of these studies, they highlight the importance of protecting our patients with cancer who are at an increased risk of serious COVID-19 infection at any age. ${ }^{8}$

\section{Prescreening and Screening Approaches}

In an effort to control the community spread of the virus, social distancing has been recommended globally. To help support this effort, healthcare facilities have implemented interventions to prescreen and screen their patients for symptoms suggestive of COVID-19 infection. In many cancer centers, prescreening is performed via phone or digital applications 1 or 2 days before the patient's upcoming visit. These screenings evaluate for symptoms such as a new or worsening cough within the past 14 days, shortness of breath, muscle aches, or fever, and may also include assessment of exposure risk, including travel history or exposure to a COVID-19-positive individual. If any concerning symptoms are reported, these are further clarified to determine whether patients need to be evaluated, monitored at home, or referred to the emergency department (ED).

A unique challenge in the cancer population is that many patients undergoing cytoreductive therapy, and especially those with lung disease, may experience similar symptoms as a consequence of treatment or due to their underlying disease process. Therefore, it is important to ensure that patients are appropriately assessed for other potential etiologies, including other infections (eg, blood cultures in the management of patients with neutropenic fever).

If patients have mild symptoms, they are encouraged to stay at home with close monitoring. If possible, evaluation via telehealth should be coordinated to decrease the risk of potential exposure to other patients and staff. Many institutions have also established screening clinics that are separated from other clinical areas to reduce the risk of exposure to asymptomatic patients with cancer who are seeking care. These screening clinics allow for dedicated staff to evaluate and potentially test symptomatic patients for SARS-CoV-2. Some cancer centers have also created off-site testing locations, including drive-throughs, which allow for efficient testing of symptomatic patients without increasing exposure risk. If patients are unstable and are referred to the ED, it is recommended that a separate area within or outside the ED is designated for vulnerable populations, including patients with cancer. The ability to erect these clinics depends on the availability of rapid testing capabilities, supply of appropriate personal protective equipment (PPE), and space, which is limited in areas already seeing a surge of COVID-19 cases.

If symptomatic patients present to the cancer center for treatment after a negative prescreening assessment, they must be provided with a mask and directed to a screening clinic for evaluation and potential testing before moving forward with any cancer-directed therapy. Cancer centers should develop strategies for the management of these symptomatic patients, including rescheduling of nonessential visits. Thus, it is important that cancer centers determine what constitutes an "essential visit" and develop infection prevention guidance to ensure appropriate management of such patients should they require an in-person visit. Although nonessential visits and therapy should be curtailed, there will continue to be a requirement for life-saving and lifeprolonging interventions in COVID-19-positive patients. For patients who are symptomatic or have tested positive for COVID-19, some cancer centers have established cohorted treatment areas. Grouping this population enables necessary and clinically appropriate therapy to continue, at the physician's discretion, with lower risk of exposure to others. In addition, patients who are COVID-19-positive or under evaluation for COVID-19 may receive their care in designated units when they are admitted to the hospital.

\section{Caregivers}

Because patients with cancer who are receiving therapy are considered to be a high-risk population, their exposure should be limited to their own family and cohabiters. Data suggest that confirmed cases of COVID19 have had close contact with other symptomatic cases. ${ }^{9}$ Therefore, if the patients have a caregiver who may be exhibiting symptoms, it is essential that the caregiver socially distance themselves, immediately seek care, and potentially receive testing for COVID-19. It is important to educate caregivers that while they may not themselves be at a high risk for complications from COVID-19, their close relationship with a patient with active cancer can put the patient at risk. As an extension of this concept, many if not all cancer centers have implemented limited or no visitor policies, with exceptions for patients with disabilities or those at the end of life. In an effort to include caregiver support during patient visits and treatments, telephone or video communication with caregivers should be accommodated. Cancer center 
policies can be communicated to patients and visitors via letters and emails, and during phone screening to set appropriate expectations.

\section{Telemedicine}

A retrospective study of patients with cancer in China showed that recurrent hospital visits and admissions were potential risk factors for COVID-19. ${ }^{7}$ As this information supports the tenet of social distancing, many institutions have expanded their telemedicine programs to allow for transition of in-person visits to telephone encounters or video visits. ${ }^{10}$ With broadened access criteria from Centers for Medicare \& Medicaid Services for telehealth, remote visits via video allow for both follow-up care for established patients and initial consultations for new patients. ${ }^{11}$ Support services, such as palliative care, dietitian services, and psycho-oncology, can also be delivered via telemedicine to continue providing comprehensive cancer care.

\section{Changes in Cancer Care}

Because many institutions are required to limit elective procedures and surgeries, a multidisciplinary discussion should occur in planning the optimal treatment course for any patient. Patients who require diagnostic biopsies via aerosol-producing procedures, such as endoscopies, endoscopic ultrasounds, or bronchoscopies, may undergo CT-guided biopsies if possible. Molecular testing and liquid biopsies may also be weighed for some circumstances, with the caveat that some of these techniques are still being validated. For patients being considered for systemic therapy for cancer or who need to continue treatment, several strategies can be used. If possible, systemic therapy can be administered with alternative dosing schedules to allow the patient to have fewer in-person visits. For infusional therapies that have equivalent oral formulation, changing to oral oncolytics can also be considered to reduce the frequency of inperson visits. For patients on continuous infusions or those who require growth factor support, protocols that allow for pump disconnect or administration of growth factors at home may be implemented. Some therapies can also be administered at home through home care nurses, including luteinizing hormone-releasing hormone agonists for patients with prostate or breast cancer. Although no significant data are available to suggest an inpatient hospitalization puts patients at higher risk than recurrent outpatient visits, given probable nearterm inpatient bed demand, an NCCN toolkit is available to assist in shifting traditionally inpatient chemotherapy regimens into the outpatient setting, primarily for patients with hematologic malignancies. ${ }^{12}$ Patients whose treatment plan includes radiation therapy may be evaluated for short-course radiation therapy or hypofractionated radiation therapy if this is an appropriate alternative. If restaging scans are routinely obtained to evaluate treatment response, increased time interval between scans or the use of biochemical markers in lieu of scans can be considered.

\section{Hematologic Malignancies and Stem Cell Transplant Considerations}

Similar to the considerations for solid tumor malignancies, the care of patients with hematologic malignancies is focused on delivering only the essential elements necessary to achieve high-quality care. Unique to the malignant hematology population are prolonged hospitalizations for induction chemotherapy in acute myeloid leukemia and acute lymphoblastic leukemia. Given that these are life-threatening conditions and chemotherapy can be curative, it is not recommended that these therapies be delayed or seen as elective. However, asymptomatic patients who will receive chemotherapy that results in significant and prolonged immunosuppression should be considered for COVID-19 testing before therapy is started. Impending blood product shortages will require strict adherence to guidelines for single-unit red cell transfusions in patients with a hemoglobin level $<7 \mathrm{~g} / \mathrm{dL}$ and platelet transfusions for values $<10,000 \mathrm{~K} / \mathrm{uL}$.

Also unique to this population is the use of autologous or allogeneic stem cell transplant. Recommendations for proceeding with transplantation in the setting of limited hospital resources, blood product shortages, and donor stem cell procurement challenges have resulted in thoughtful consideration of which disease indications require urgent transplant.

However, even in patients whose disease warrants immediate action, processes for obtaining donor stem cells have been modified. Due to global transmission and spread of COVID-19 and associated travel restrictions, the National Marrow Donor Program requires all transplant centers to receive and cryopreserve unrelated donor products before starting recipient-conditioning chemotherapy. Additionally, because many centers have limited their performance of nonemergent surgical procedures, peripheral blood mobilized stem cell products are preferred over bone marrow grafts requiring surgical harvest, except in circumstances where data support improved survival with the use of bone marrow graft over peripheral blood graft.

\section{Care of the Oncology Patient With COVID-19}

No clear guidelines exist regarding when a patient with cancer who has tested positive for COVID-19 can resume therapy. At the time of writing, the $\mathrm{CDC}$ has recommended a test-based strategy for immunocompromised patients that involves 2 consecutive negative nasopharyngeal swabs 
collected $\geq 24$ hours apart and resolution of fever and respiratory symptoms. ${ }^{13}$ (However, the CDC also notes that "testing guidance is based upon limited information and is subject to change as more information becomes available.") This is the preferred approach among centers with access to testing. A non-test-based strategy would include at least 3 days since resolution of symptoms and at least 7 days since symptoms first appeared. The latter strategy leaves many questions unanswered, including the length of viral shedding and the timeline for when PPE can be safely discontinued.

In addition, there are patients with cancer who have COVID-19 who may need to continue their cancer treatment, such as those receiving radiation therapy for whom treatment interruption may diminish the local control benefit of therapy. Care of these patients must be managed carefully, with prior coordination between the environmental services, treating team, and operational unit to allow for a controlled and safe environment for all patients. COVID-19-positive patients should wear a mask and be treated in a separate area from other patients, and their visits may be temporally separated from other patients' appointments. Minimal staff members should be involved in the treatment day, and always with appropriate PPE. The treatment area should be cleaned and disinfected per the COVID-19 guidelines available by the US Environmental Protection Agency. ${ }^{14}$

\section{Healthcare Worker Safety}

As the frontline caregivers for patients with COVID-19, healthcare workers are considered a high-risk population. Approximately $3.8 \%$ to $10 \%$ of the confirmed COVID-19-positive cases in China, Italy, and Spain were healthcare workers. ${ }^{3-5}$ The optimal approach to protecting healthcare workers in the face of a worldwide shortage of PPE has been addressed by public health agencies, with some nuanced differences in approach. The WHO recommends contact and droplet precautions (including eye protection) for the management of suspected or confirmed COVID-19 cases. As of April 1, 2020, the CDC recommends use of an N95 or higher respirator, but use of a facemask is an acceptable alternative (if a respirator is unavailable) when providing routine care to patients with known or suspected COVID-19. ${ }^{15}$ The PPE approach for suspected or confirmed COVID-19 cases may vary at each institution based on guidance from local public health jurisdiction and supply.

When performing aerosol-generating procedures on patients with confirmed or suspected COVID-19, airborne (using N95 or higher respirator), contact, and droplet precautions (including eye protection) are recommended.

The WHO recommends strategies to maximize PPE use to protect healthcare workers while minimizing the need for PPE in several ways, including (1) use of telemedicine for initial evaluation, (2) having only essential healthcare workers enter patient rooms, and (3) bundling activities to minimize the number of times a room is entered. ${ }^{16}$ Screening clinics not only provide a dedicated area for evaluation of patients with potential COVID-19 but also allow for PPE conservation by staff. The FDA issued an Emergency Use Authorization for the Battelle CCDS Critical Care Decontamination System for decontaminating compatible N95 or N95-equivalent respirators, which may assist with N95 shortages in areas where this technology is available. ${ }^{17}$

Recently, the CDC developed a PPE burn rate calculator for healthcare facilities to "plan and optimize the use of PPE for response to COVID-19."18 A muchdiscussed topic in the midst of PPE shortage has been whether universal or extended use of surgical masks in the clinical setting should be recommended. Although the CDC has not released guidance on this issue for healthcare settings, on April 3, 2020, it recommended the use of cloth face coverings in public settings where other social distancing measures are difficult to maintain. Many cancer centers have already adopted this policy due to the difficulty in sustaining a healthy distance of 6 feet in clinical care settings. If such an approach is implemented, it is important to carefully consider the implications on PPE supply and develop mitigation strategies.

In addition to PPE use, several other measures can protect healthcare workers, including the early adoption of travel restrictions, arranging meetings over teleconference, and establishing clear guidelines on when to stay at home and when to return to work. Healthcare workers with any concerning symptoms or exposure history should self-isolate and not report to work. Establishing screening tools, daily questionnaires, and/or temperature checks to assess symptomatic healthcare workers decreases the risk of exposure from potentially COVID-19-positive individuals. Institutions should develop COVID-19 hotlines in collaboration with occupational health services to triage symptomatic healthcare workers to screening clinics for rapid testing.

If a healthcare worker is exposed to a COVID-19-positive patient without appropriate protection, they should contact the occupational health office of their institution for guidance on local policy. Investigation of exposure history should also be performed by occupational health to identify any other individuals who may be at risk. Healthcare workers who are unable to present to clinical areas due to mild symptoms can be encouraged to participate in telemedicine activities to assist in the management of patients who have respiratory symptoms. When possible, telecommuting for all employees should be encouraged, with limited on-site staff who may rotate on a daily basis to reduce their exposure risk. A systematic rotating staff team system may also enable streamlined tracing of contacts, if an employee becomes COVID-19-positive. 
Institutions participating in telecommuting should make "remote work tools" available to their employees to allow telecommuting to be effective.

For healthcare providers who have suspected or confirmed COVID-19, it is important to adhere to stay-athome and return-to-work guidelines. Test-based and non-test-based strategies are available to clear them to return to work. ${ }^{19}$

- Test-based strategy includes "at least 2 consecutive nasopharyngeal swab specimens collected $\geq 24$ hours apart for SARS-CoV-2 PCR (total of 2 negative specimens) and resolution of fever without the use of medications and improvement of respiratory symptoms."

- Non-test-based strategy includes returning to work "at least 3 days following resolution of fever without the use of medications and improvement in respiratory symptoms and at least 7 days have passed since symptoms first appeared."

At times of surge, it is likely that these guidelines will have to be revisited to allow for adequate workforce support on the front lines while still prioritizing the safety of patients and healthcare workers. The FDA approved the first antibody test for SARS-CoV-2 on April 2, 2020; as serologic tests become available, they will have important implications in helping to identify an immune workforce to care for patients with COVID-19.

\section{Wellness Resources}

Frontline healthcare workers in China managing patients with COVID-19 reported anxiety (50.4\%), insomnia $(34 \%)$, and distress $(71.5 \%) .{ }^{20}$ Because of concerns regarding shortages of PPE and increased risk of infections for self, family, and patients, it is important for healthcare workers to have the resources to assist with wellness and mental health. As we encourage social distancing, it is important that it not lead to social isolation. NCCN recently published guidance for self-care and stress management for oncology healthcare professionals. ${ }^{21}$ Recommendations include taking brief breaks during shifts and making time to do what brings one joy. A balanced diet, regular activity, and adequate sleep are also important. Consultation with a mental health provider or availability of virtual visits for meditation and crisis intervention teams may also provide some comfort during this challenging time.

Similar support services can also be useful for patients who may be experiencing an emotional toll from delaying care, the risk of COVID-19 exposure on their health, and keeping abreast of rapid shifts in how their care is delivered. Cancer centers have adapted practice by leveraging virtual technology to continue offering online support groups, providing telehealth for psychotherapy, and expanding a digital footprint for educational and experiential programming. However, the full breadth of supportive services may be diminished. The role of palliative care services via telemedicine has also been shown to provide patients with additional support at home and can be used to address symptoms and end of end-of-life issues during the pandemic.22 NCCN's self-care guide for patients can be an important resource as patients navigate their emotions at this time. ${ }^{23}$

\section{Communication With Patients and Healthcare Workers Regarding Safety}

Keeping all healthcare team members, caregivers, and patients informed during a rapidly evolving situation such as COVID-19 should be a primary focus of leadership. Regular meetings via daily huddles as well as email communications with real-time updates that are pertinent and meaningful will provide some reassurance in a time of uncertainty. Weekly or biweekly town hall meetings may also provide a venue to address questions and inform the cancer center community of updates and challenges. Regularly updated internal websites featuring guidelines and policies regarding COVID-19 and

\section{Table 1. Recommendations for Oncology Practices During COVID-19 \\ Patient Safety}

Prescreen and screen for COVID-19 symptoms and exposure history via telephone calls or digital platforms

Develop screening clinics to allow for patients with symptoms to be evaluated and tested in a dedicated unit with dedicated staff

Convert in-person visits to telemedicine visits when possible

Institute limited or no visitor policy

Limit surgeries and procedures to only essential, urgent, or emergent cases

Consider alternative dosing schedule to allow for fewer in-person visits to the cancer center and/or the infusion center

Switch therapy to oral oncolytics if equivalent formulation of infusional therapy is available

Transition outpatient care to care at home whenever possible (eg, pump disconnection, administration of growth factors, hormone therapy)

Increase interval between scans or use biochemical markers in lieu of scans

Provide resources for wellness and stress management for patients

\section{Healthcare Worker Safety}

Assure appropriate personal protective equipment (PPE) per guidelines

Create a centralized resource or website to communicate the recommendations to the healthcare workers as guidelines around PPE and workflows change

Implement daily screening tools and/or temperature checks

Telecommute when possible, with limited onsite staff participating in rotations on a daily basis

Establish clear stay-at-home and return-to-work guidelines

Provide resources for wellness and stress management for healthcare workers 
information on wellness, childcare, PPE, workflows, and clinical algorithms will be an invaluable resource for healthcare workers. Developing and distributing a document with frequently asked questions will also provide patients and their families with a sense of ease.

\section{Conclusions}

At a time of crisis, the prime foci for every institution are protecting the well-being of patients and healthcare workers while continuing to provide a nurturing and safe environment to work and care for patients. Although various measures can be implemented in providing the best possible cancer care in a safe manner, it is important to acknowledge the rapidly evolving nature of the COVID-19 pandemic and the uncertainties that come with it (Table 1). The distressing global experience with COVID-19 limited, and in some cases devastated, the healthcare delivery systems we have relied on to provide safe and effective care to our patients. Nonetheless, our healthcare community remains dedicated, resilient, and adaptable. Most of all, the commitment we have to the patients we serve will continue to guide our response during these extraordinarily challenging times. With careful evaluation and modification of our approach, we can continue to provide our patients with effective and compassionate care without sacrificing the health and safety of our teams, colleagues, and families.

Submitted April 1, 2020; accepted for publication April 3, 2020.

Publication: This is an invited article from the NCCN Best Practices Committee.

Disclosures: Dr. Fonesca disclosed that he has served as a consultant for Amgen, Bristol Myers Squibb, Celgene, Takeda, Bayer, Janssen, Novartis, Pharmacyclics, Sanofi, Merck, Juno, Kite, Aduro, OncoTracker,

GlaxoSmithKline, and AbbVie, and is on the Scientific Advisory Board for Adaptive Biotechnologies and OncoTracker. The remaining authors have disclosed that they have no financial interests, arrangements, affiliations, or commercial interests with the manufacturers of any products discussed in this article or their competitors.

Correspondence: Pelin Cinar, MD, MS, Division of Medical Oncology, Department of Medicine, UCSF Helen Diller Family Comprehensive Cancer Center, 550 16th Street, 6th Floor, Box 3211, San Francisco, CA 94143. Email: pelin.cinar@ucsf.edu.

\section{References}

1. WHO. WHO Director-General's opening remarks at the media briefing on COVID-19 - 11 March 2020. Accessed March 27, 2020. Available at: https://www.who.int/dg/speeches/detail/who-director-general-s-opening-remarks-at-the-media-briefing-on-covid-19---11-march-2020

2. Johns Hopkins University. Coronavirus COVID-19 global cases by the Center for Systems Science and Engineering (CSSE) at Johns Hopkins University. Accessed March 29, 2020. Available at: https://www.arcgis.com/apps/opsdashboard/index.html\#/ bda7594740fd40299423467b48e9ecf6

3. The Novel Coronavirus Pneumonia Emergency Response Epidemiology Team. Vital surveillances: the epidemiological characteristics of an outbreak of 2019 novel coronavirus diseases (COVID-19) - China, 2020. CCDC Weekly 2020;2:113-122. Available at: http://weekly.chinacdc.cn/ en/article/id/e53946e2-c6c4-41e9-9a9b-fea8db1a8f51

4. Instituto Superiore di Sanita. Integrated surveillance of COVID-19 in Italy. Accessed March 28, 2020. Available at: https://www.epicentro.iss.it/ coronavirus/bollettino/Infografica_28marzo\%20ENG.pdf

5. Red Nacional de Vigilancia Epidemiológica. Informe sobre la situación de COVID-19 en España: Informe COVID-19 no 17. 27 de marzo de 2020. Accessed March 29, 2020. Available at: https://www.isciii.es/QueHacemos/Servicios/VigilanciaSaludPublicaRENAVE/EnfermedadesTransmisibles/Documents/INFORMES/Informes\%20COVID-19/Informe\%20n-\% 2017.\%20Situación\%20de\%20COVID-19\%20en\%20España\%20a\% 2027\%20marzo\%20de\%202020_revisado.pdf

6. Liang W, Guan W, Chen R, et al. Cancer patients in SARS-CoV-2 infection: a nationwide analysis in China. Lancet Oncol 2020;21:335-337.

7. Yu J, Ouyang W, Chua MLK, Xie C. SARS-CoV-2 transmission in patients with cancer at a tertiary care hospital in Wuhan, China [published online March 25, 2020]. JAMA Oncol, doi: 10.1001/jamaoncol. 2020.0980

8. CDC. Implementation of mitigation strategies for communities with local COVID-19 transmission. Accessed March 27, 2020. Available at: https:// www.cdc.gov/coronavirus/2019-ncov/downloads/community-mitigationstrategy.pdf

9. CDC. How COVID-19 spreads. Accessed April 3, 2020. Available at: https://www.cdc.gov/coronavirus/2019-ncov/prevent-getting-sick/howcovid-spreads.html

10. Ueda M, Martins R, Hendrie PC, et al. Managing cancer care during the COVID-19 pandemic: agility and collaboration toward a common goal. J Natl Compr Canc Netw 2020;18:366-369.

11. CMS. General provider telehealth and telemedicine tool kit. Accessed March 27, 2020. Available at: https://www.cms.gov/files/document/ general-telemedicine-toolkit.pdf

12. NCCN Best Practices Committee Infusion Efficiency Workgroup. Toolkit providing oncology treatments in the outpatient setting. Accessed April 1 2020. Available at: https://www.nccn.org/covid-19/pdf/NCCN_Toolkit Updated_for_COVID-19.pdf

13. CDC. Discontinuation of in-home isolation for immunocompromised persons with COVID-19 (interim guidance). Accessed March 27, 2020. Availabe at: https://www.cdc.gov/coronavirus/2019-ncov/hcp/endingisolation.html

14. EPA. List N: disinfectants for use against SARS-CoV-2. Accessed March 26, 2020. Available at: https://www.epa.gov/pesticide-registration/listn-disinfectants-use-against-sars-cov-2

15. CDC. Interim infection prevention and control recommendations for patients with suspected or confirmed coronavirus disease 2019 (COVID-19) in healthcare settings. Accessed April 1, 2020. Available at: https://www.cdc.gov/coronavirus/2019-ncov/infection-control/controlrecommendations.html?CDC_AA_refVal=https\%3A\%2F\%2Fwww.cdc. gov\%2Fcoronavirus\%2F2019-ncov\%2Fhcp\%2Finfection-control.html

16. WHO. Rational use of personal protective equipment (PPE) for coronavirus disease (COVID-19) 2020. Accessed April 1, 2020. Available at: https:// apps.who.int/iris/bitstream/handle/10665/331215/WHO-2019-nCovIPCPPE_use-2020.1-eng.pdf

17. Emergency Use Authorization (EUA) for the emergency use of the Battelle CCDS Critical Care Decontamination System. Accessed March 31, 2020 Available at: https://www.fda.gov/media/136529/download

18. CDC. Personal protective equipment (PPE) burn rate calculator. Accessed March 27, 2020. Available at: https://www.cdc.gov/coronavirus/2019ncov/hcp/ppe-strategy/burn-calculator.html

19. CDC. Return to work criteria for HCP with confirmed or suspected COVID19. Accessed 26, 2020. Available at: https://www.cdc.gov/coronavirus/ 2019-ncov/healthcare-facilities/hcp-return-work.html

20. Lai J, Ma S, Wang Y, et al. Factors associated with mental health outcomes among health care workers exposed to coronavirus disease 2019 [published online March 23, 2020]. JAMA Netw Open, doi: 10.1001/jamanetworkopen.2020.3976

21. NCCN. Self-care and stress management during the COVID-19 crisis: toolkit for oncology health care professionals. Accessed March 26, 2020. Available at: https://www.nccn.org/covid-19/pdf/Distress-ManagementClinician-COVID-19.pdf

22. Calton $B A$, Rabow MW, Branagan $L$, et al. Top ten tips palliative care clinicians should know about telepalliative care. J Palliat Med 2019;22:981-985.

23. NCCN. Self-care \& distress management during the COVID-19 pandemic Accessed April 1, 2020. Available at: https://www.nccn.org/covid-19/pdf/ NCCN_Patient_Self_Care_COVID-19.pdf 